\title{
Analysis of the microstructure of superconducting YBCO foams by means of AFM and EBSD
}

\author{
Michael Rudolf KOBLISCHKA ${ }^{a,{ }^{*}}$, Anjela KOBLISCHKA-VENEVA ${ }^{a}$, \\ E. S. REDDY ${ }^{b, c}$, Gregor J. SCHMITZ ${ }^{c}$ \\ ${ }^{a}$ Institute of Experimental Physics, Saarland University, P. O. Box 151150, D-66041 Saarbrücken, Germany \\ ${ }^{b}$ ACCESS, Intzestrasse 5, 52072 Aachen, Germany \\ ${ }^{c}$ Nanonouvelle Pty Ltd., Marcoola QLD 4564, Australia
}

Received: June 12, 2014; Accepted: July 23, 2014

(CThe Author(s) 2014. This article is published with open access at Springerlink.com

\begin{abstract}
YBa}_{2} \mathrm{Cu}_{3} \mathrm{O}_{y}$ (YBCO) foam samples show an open, porous foam structure, which may have benefits for many applications of high- $T_{\mathrm{c}}$ superconductors. As the basic material of these foams is a pseudo-single crystalline material with the directional growth initiated by a seed crystal similar to standard melt-textured samples, the texture of YBCO is a very important parameter. Therefore, we analysed the local texture and grain orientation of the individual struts forming the foam by means of atomic force microscopy (AFM) and electron backscatter diffraction (EBSD). Due to the processing route starting with $\mathrm{Y}_{2} \mathrm{BaCuO}_{5}(211)$, a two-phase analysis must be performed, so a high surface quality is necessary to enable an automated EBSD scan. Good quality Kikuchi patterns were obtained from both the YBCO and 211 phases. We found an inhomogeneous distribution of the residual 211 particles, which are mainly randomly oriented and have sizes ranging between $200 \mathrm{~nm}$ and $15 \mu \mathrm{m}$. In contrast to this, the YBCO matrix shows a dominating orientation with cracks with a typical distance of $1-10 \mu \mathrm{m}$. Furthermore, the analysis of strut cross-sections reveals that the entire strut is converted to the YBCO phase.
\end{abstract}

Keywords: high- $T_{\mathrm{c}}$ superconductors; foam; microstructure; atomic force microscopy (AFM); electron backscatter diffraction (EBSD); orientation

\section{Introduction}

The development of $\mathrm{YBa}_{2} \mathrm{Cu}_{3} \mathrm{O}_{y} \quad$ (YBCO) superconducting foams presented in Refs. [1-6] is an important step forward to obtain large, but lightweight superconducting bulks, which will be important for several applications like the all-electric plane [7] or applications in space [8,9]. Metallic foams also play an important role as shock-absorbers etc. [10]. However,

\footnotetext{
* Corresponding author.

E-mail: m.koblischka@mx.uni-saarland.de
}

only recently, the specific properties of these samples are discussed in more detail [11,12]. Additionally, there are some technical advantages of such a sample configuration: the open structure of the foams enables an easy oxygenation of a bulk sample which otherwise would take extremely long [13], and furthermore it is possible to send the coolant (i.e., liquid $\mathrm{N}_{2}$ ) directly through the sample. All these make the foams interesting candidates for applications, but further detailed analysis of the resulting foam properties is required.

While several types of characterization measurements like magnetization, resistance, X-ray 
diffraction and texture analysis, neutron diffraction, polarized light and scanning electron microscopy (SEM) were carried out on the foam samples [1-6], a detailed analysis of the resulting microstructure of the individual struts is still missing in the literature. The character of the resulting microstructure is especially interesting as the superconducting foams are prepared starting from a $\mathrm{Y}_{2} \mathrm{BaCuO}_{5}$ (211)-coated polyurethane foam. The preparation employs a burnout of the organic materials involved, then followed by a conversion of the 211 phase into YBCO [1,2]. As a result, 211 particles will be formed in the superconducting matrix, and their behavior may directly influence the growth of the superconducting matrix. This was already studied earlier by us by means of electron backscatter diffraction (EBSD) $[14,15]$ and others employing neutron diffraction $[16,17]$. The development of the EBSD technique now enables a spatial resolution on ceramic materials which is similar to the atomic force microscopy (AFM) topography measurement [18,19]. Therefore, we present here a thorough investigation of the microstructure of such YBCO foam struts by means of AFM and EBSD.

\section{Experimental procedure}

\section{1 Preparation of foams}

The superconducting foams were prepared at ACCESS, Aachen, following a two-step process [1-5]. As the preparation route is very important for the understanding of the resulting microstructure, we repeat here the most important points. In the first step, a porous 211 "skeleton" foam was made by an established ceramic foam fabrication process as follows. A polyurethane foam with the desired porosity was impregnated in a 211 slurry, formed by mixing commercially available 211 powder $(1-5 \mu \mathrm{m}$ grain size) in a water-based solution with $5 \mathrm{wt} \%$ polyvinyl alcohol as binder. A thermal process followed such that the organic components (polyvinyl alcohol and polyurethane) were burnt off by slow heating at $50{ }^{\circ} \mathrm{C} / \mathrm{h}$ to $600{ }^{\circ} \mathrm{C}$ and dwelling during $6 \mathrm{~h}$, and the resulting 211 ceramic was densified by further heating at $150{ }^{\circ} \mathrm{C} / \mathrm{h}$ to $1100{ }^{\circ} \mathrm{C}$. In a second step, the formed 211 foam was converted into a single-domain YBCO foam by an infiltration process followed by a top-seeding growth method, which is commonly used for the growth of melt-textured single domains. In this process a liquid phase source, formed by a 1:1 mixture of barium and copper oxides and additional YBCO powder, was placed under the 211 foam, and the entire assembly was heated up above the peritectic temperature of $1010{ }^{\circ} \mathrm{C}$. The YBCO phase decomposed into solid 211 and liquid phase, which infiltrated into the 211 skeleton by capillarity. The infiltrated 211 foam was then slowly cooled down $\left(0.3{ }^{\circ} \mathrm{C} / \mathrm{h}\right)$ through the peritectic temperature with a $\mathrm{NdBa}_{2} \mathrm{Cu}_{3} \mathrm{O}_{y}$ seed crystal centered on top of a $\mathrm{Y}_{2} \mathrm{O}_{3}$ fabric, resulting in the growth of a single-crystalline YBCO foam.

\section{2 Sample surface preparation}

Since the as-grown surfaces of the samples were usually too rough to achieve good scanning results, the samples were polished prior to scanning, either dry from $12 \mu \mathrm{m}$ to $0.5 \mu \mathrm{m}$ diamond paper or wet from 320 grain $\mathrm{SiO}$ paper to 4000 grain $\mathrm{SiO}$ paper and then from $3 \mu \mathrm{m}$ diamond polishing solution down to $40 \mathrm{~nm}$ colloidal silica suspension [20]. After that, the samples were cleaned for several minutes in acetone in an ultrasonic bath and then for several minutes in an ethanol bath. Both surface preparation methods served well for the AFM/STM (scanning tunneling microscopy) measurements, as well as for magnetooptic and EBSD analysis [21].

\section{3 Scanning probe topography measurements}

We employed Digital Instruments Nanoscope III and IV controllers in AFM mode and STM mode at ambient conditions. For comparison, AFM scans were performed in contact mode and tapping mode using micro-machined, doped Si-cantilevers (type PPP, Nanoworld Services GmbH, Erlangen, Germany). A Q-control unit was used to improve the signal-to-noise ratio in the tapping mode [22]. STM scans were done using cut Pt/Ir-tips [23]. As both AFM and STM shared the same scanner but employed different types of tips, this enabled to exclude influences of the cantilevers. Any effects of the measurement direction were excluded by employing different scan directions in both AFM and STM measurements. The tunnelling voltages ranged between $0.1 \mathrm{~V}$ and $0.5 \mathrm{~V}$, sometimes up to $1 \mathrm{~V}$. The tunnel currents were for most images below $1 \mathrm{nA}$.

\subsection{Electron backscatter diffraction}

The EBSD system employed here consisted of a JEOL 
SEM (JSM 7000F) equipped with a TSL OIM analysis unit [24]. The Kikuchi patterns were generated at an acceleration voltage of $20 \mathrm{kV}$, and were recorded by means of a DigiView camera system, allowing a maximum recording speed in the order of $0.05 \mathrm{~s} /$ pattern. The time employed in the case of a multi-phase scan was much longer, in the order of $0.4 \mathrm{~s} /$ pattern, as a higher image quality/confidence index was required. To produce a crystallographic orientation map, the electron beam was scanned over a selected surface area and the resulting Kikuchi patterns were indexed and analysed automatically (i.e., the Kikuchi bands were detected by means of the software). An image quality (IQ) parameter and a confidence index (CI) were recorded for each Kikuchi pattern. The dimensionless IQ parameter was the sum of the detected peaks in the Hough transform employed in the image recording; the CI value yielded information about how exact the indexation was carried out. The CI value ranged between 0 and 1 [24]. Based on the analysis of the recorded CI value, a multi-phase analysis was realised. A detailed description of the measurement procedure can be found in Refs. [19,25].

\section{Results and discussion}

We have analysed in detail single struts broken from a big foam piece by means of AFM and EBSD. Figure 1(a) illustrates the superconducting foam and the selected places for taking out the individual struts as marked by the blue arrows. All samples for investigation were selected from the middle section of the original foam piece, which measures $5 \mathrm{~cm} \times 2 \mathrm{~cm} \times$ $2 \mathrm{~cm}$. At the positions (1) and (2) close to the $\mathrm{Y}_{2} \mathrm{O}_{3}$

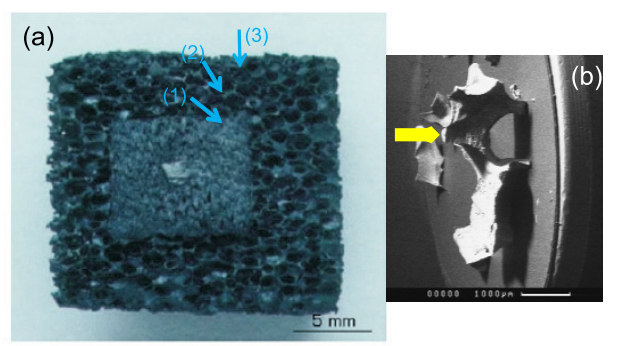

Fig. 1 (a) View of the entire foam piece, together with the fabric and the seed crystal. The arrows define the locations (1), (2) and (3) where struts were broken out for the investigations. (b) SEM image of the mounted foam struts for EBSD investigations under an angle of $70^{\circ}$. The yellow arrow points to the investigated strut cross-section. fabric which is placed under the seed crystal, we expect still a good texture of the superconducting matrix, while at a place far outside (3) the situation may be different. Figure 1(b) shows the prepared foam piece for the EBSD cross-section analysis. Note that the sample was polished without embedding the pieces in an additional mounting resin. The electron beam will hit the polished top surface of a strut as indicated by an arrow.

Figures 2(a) and 2(b) present typical AFM topography scans of the microstructure of a mechanically polished piece of such a foam strut with low magnification (scan area of $90 \mu \mathrm{m} \times 90 \mu \mathrm{m}$ ). The struts analysed here stem from positions (1) and (2). The sketch indicates the orientation of the strut surface with respect to the entire foam piece. As we cannot distinguish the $a$ and $b$ axes here, we just mention the $a$-direction as it is common for bulk YBCO samples [13]. A large number of 211 particles are found here on the sample surface. A detailed size analysis reveals that the majority of the 211 particles are tiny with dimensions of $100-200 \mathrm{~nm}$, whereas the larger ones have sizes ranging between $1 \mu \mathrm{m}$ and $15 \mu \mathrm{m}$. Furthermore, the distribution of the 211 particles is inhomogeneous as in some places practically no 211 particles are obtained. Such areas are caused by particle pushing during the growth of the YBCO matrix and span typically up to $30 \mu \mathrm{m} \times 30 \mu \mathrm{m}$. Remarkably, concerning the 211 particle distribution, there is no difference between the various original
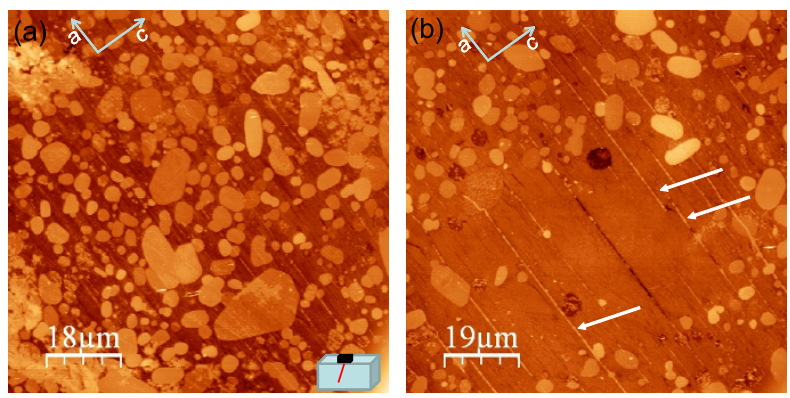

Fig. 2 AFM topography images of two locations on a foam strut. The sketch in (a) indicates the orientation of the strut with respect to the entire foam. The topography of image (a) shows a densely packed distribution of 211 particles embedded in the YBCO matrix. Clearly visible is the large amount of 211 particles on the sample surface. The particle size ranges between $1 \mu \mathrm{m}$ and $10 \mu \mathrm{m}$. (b) presents a 211 particle free area due to particle pushing. The white arrows point to cracks filled with tiny 211 particles. 
locations of the struts in the foam piece investigated here.

The YBCO matrix exhibits a large number of cracks, some of which are filled with the tiny 211 particles (Fig. 2(b), white arrows). All these cracks are oriented and run parallel to each other in $a$-direction; the $c$-axis of YBCO is oriented perpendicular to the stripes. The distance between them is ranging between $1 \mu \mathrm{m}$ and $15 \mu \mathrm{m}$. Furthermore, the direction of the cracks is not altered by the presence of the 211 particles. The origin of these stripes can only be clarified knowing also the EBSD crystallographic directions of the superconducting matrix.

Figure 3(a) presents an AFM topography image of a foam strut and Fig. 3(b) gives a 3D representation of the same data set, while Figs. 3(c) and 3(d) give data profiles together with the exact location. Clearly visible is the large amount of 211 particles on the sample surface. The particle size ranges between $1 \mu \mathrm{m}$ and $15 \mu \mathrm{m}$, which makes them not so attractive flux pinning sites. Furthermore, the 211 particles have the shape of spheres or ellipsoids. The YBCO matrix is
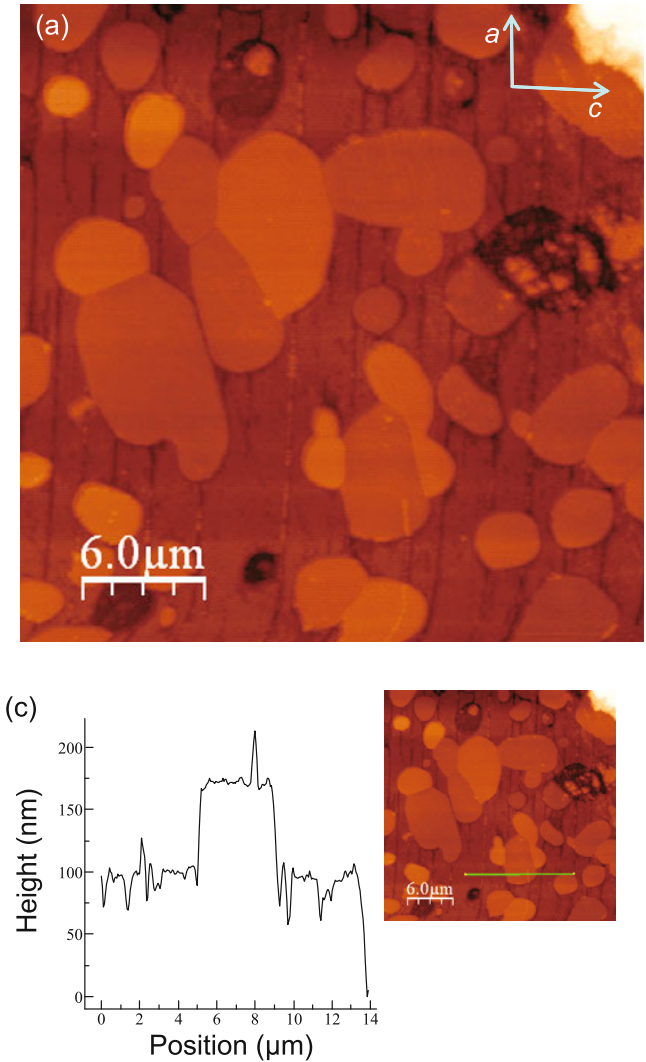

located below these particles which are much harder to be polished, and therefore form terraces, where the height difference corresponds to a unit cell of 211. In Figs. 3(c) and 3(d), the corresponding height profiles are given. Here, the cracks and the 211 plateaus become clearly visible.

Figure 4 presents the AFM topography scans with larger magnification (scan area $8 \mu \mathrm{m} \times 8 \mu \mathrm{m}$ ) on the YBCO matrix, taken at position (1). The sketch indicates the orientation of the strut surface. The cracks in the YBCO matrix have a spacing of about $1-1.5 \mu \mathrm{m}$, and the profiles reveal further details within them. Again, tiny 211 particles are located within the cracks (see also the height profile of Fig. 4(d)), but also within the superconducting matrix as indicated by arrows, which makes these particles useful for flux pinning.

In Fig. 5, we finally present an STM topography image (a) and an AFM image (b) at high magnification. Figure 5(a) shows the details around a crack in the YBCO matrix. In the STM image, the 211 particles appear bright (arrows) due to their different
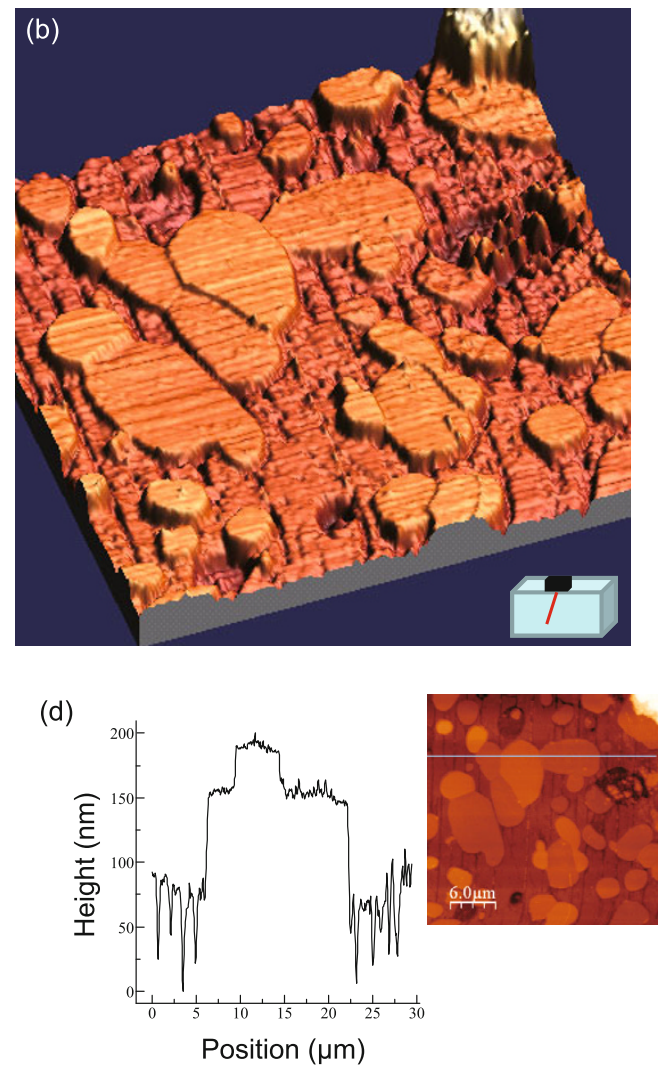

Fig. 3 (a) AFM topography image of a foam strut and (b) 3D representation of the same data set. The sketch in (b) indicates the orientation of the strut with respect to the entire foam. Clearly visible is the large amount of 211 particles on the sample surface and the resulting height differences. The superconducting YBCO matrix is located below these 211 particles, which are much harder to be polished and form terraces. (c) and (d) give selected height profiles. 


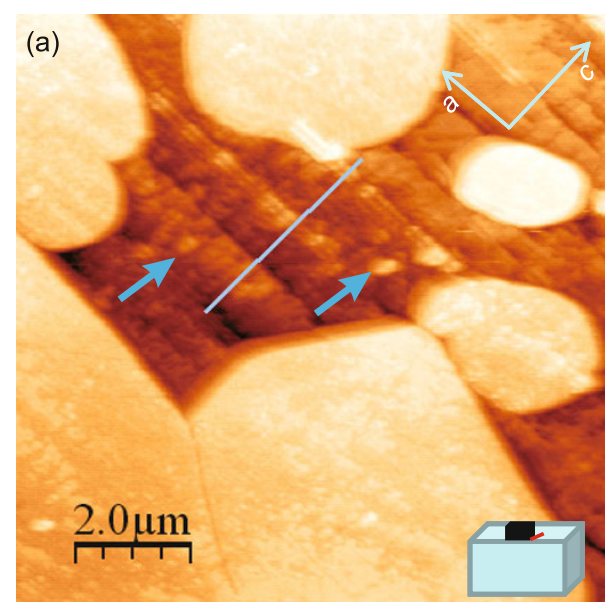

(c)

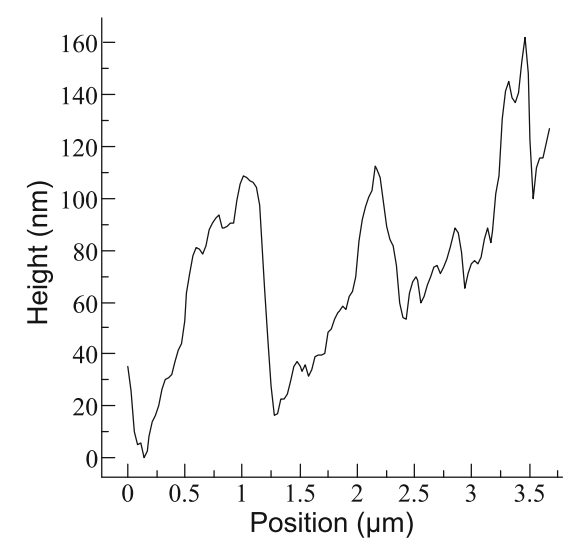

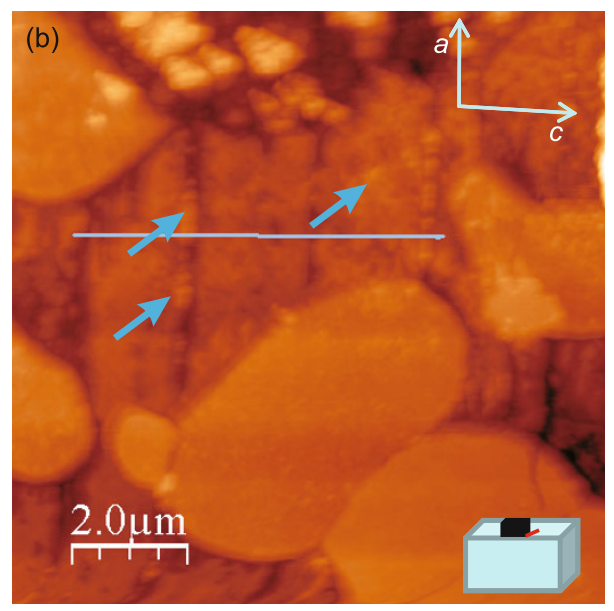

(d)

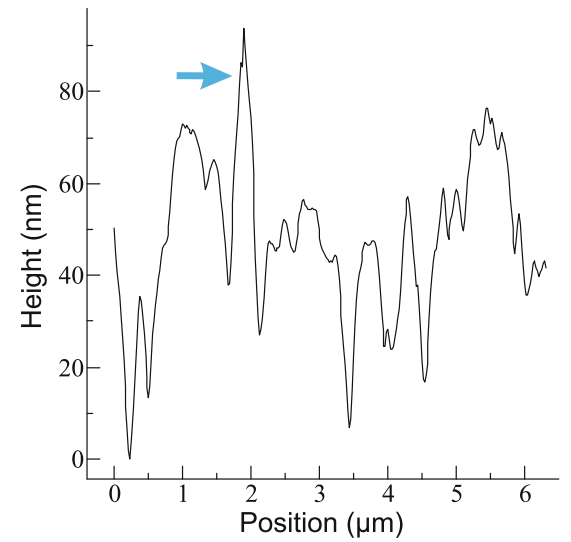

Fig. 4 (a) and (b) AFM topography scans revealing details of the YBCO matrix; (c) and (d) the corresponding height profiles. The sketches in (a) and (b) indicate the orientation of the strut with respect to the entire foam. The blue arrows point to 211 particles embedded within the YBCO matrix.
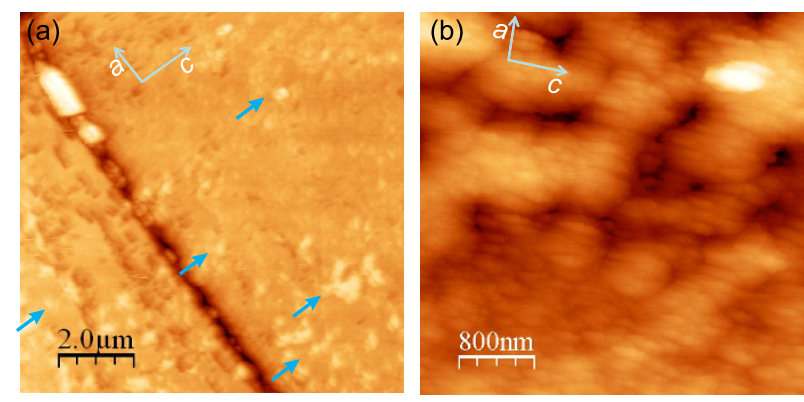

Fig. 5 (a) STM topography scan of an area close to a crack in the YBCO matrix. Here, a large number of 211 particles (blue arrows) embedded in the YBCO matrix get visible. (b) AFM topography image revealing details of the YBCO growth.

conductivity as compared to $\mathrm{YBCO}$, so it is possible to observe that many tiny 211 particles with sizes ranging between $200 \mathrm{~nm}$ and $500 \mathrm{~nm}$ do exist embedded in the YBCO matrix. On the other hand, the larger 211 particles within the crack may have a negative effect on the current flow as they cause a deeper and wider crack. Figure 5(b) reveals that the YBCO matrix consists of grains with dimensions of about $400 \mathrm{~nm} \times$ $200 \mathrm{~nm}$, which are clustered together. The long direction of these grains corresponds to the $c$-axis, and the short one is either $a$ - or $b$-oriented. The clusters form then bigger blocks with dimensions in the micrometer range.

Now, let us turn to the EBSD analysis. The advanced preparation technique [26] for polishing the strut pieces (see Fig. 1(b)) enables also to perform EBSD scans on this special type of superconducting sample as illustrated in Figs. 6 and 7. Figure 6 shows an EBSD two-phase analysis of a cross-section of a foam strut. The step size for the EBSD measurements was chosen to be $70 \mathrm{~nm}$. The cross-section surface is oriented nearly parallel to the seed crystal as indicated in the sketch below the map in Fig. 6(a). As the foam was started from a polyurethane material and then got 

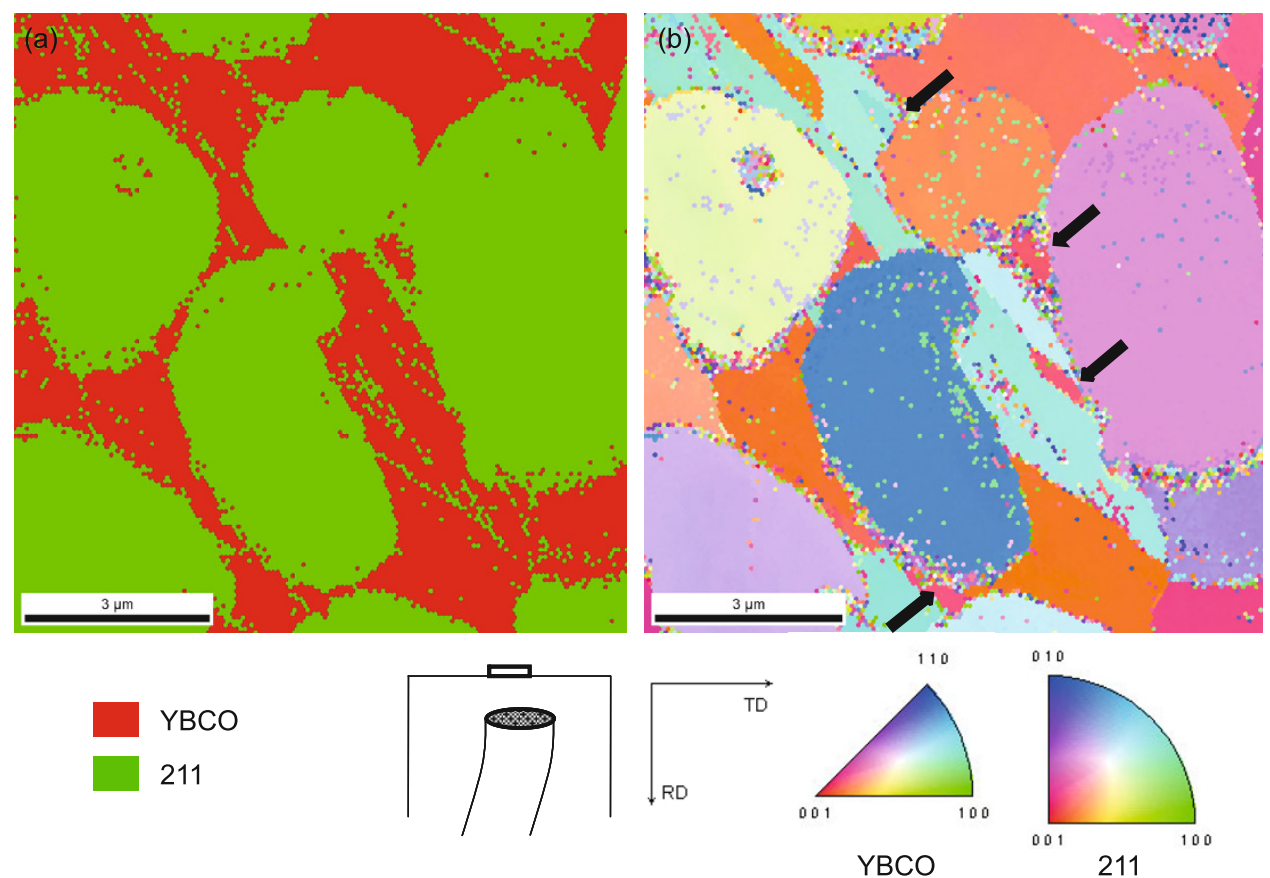

Fig. 6 (a) EBSD phase map (YBCO red and Y-211 green) and (b) inverse pole figure map in [001]-direction obtained on a cross-section of a foam strut. The color code for the crystallographic orientations is given below the image for the YBCO and 211 phases. The orientation of the strut cross-section with respect to the original foam piece is indicated in the sketch below the map. $\mathrm{RD}$ (rolling direction) and TD (transverse direction) refer to the sample reference frame. The arrows in (b) point to misoriented YBCO grains due to the presence of the large 211 grains.

covered by a slurry of 211 , it is an interesting question whether the final product contains a 211 kernel or not. This is the reason for choosing a cross-section of a strut for the EBSD analysis. Figure 6(a) represents a phase map, where YBCO is shown in red and the 211 phase in green. Figure $6(\mathrm{~b})$ is the corresponding inverse pole figure map giving the crystallographic orientation of the two phases; the color code is given in the stereographic triangles below the map. The YBCO phase is selected as tetragonal (material file from the TSL database) as in the present investigation the oxygenation state is not relevant. The EBSD analysis reveals that the strut is homogeneously converted to the YBCO phase and there is no 211 kernel residing. The YBCO phase is formed throughout the entire cross-section of the strut, not only in a surface layer. A large amount of 211 phase is present also in this configuration; the EBSD-determined total fraction is $67.9 \%$ for 211 and only $32.1 \%$ for YBCO, which is also seen in the AFM measurements. The 211 grains have clearly the shape of spheres or ellipsoids (note that the EBSD measurement is performed perpendicular to the AFM investigations as shown before). The EBSD analysis further reveals the 211 particles filling the cracks within the YBCO matrix. From Fig. 6(b), one obtains the crystallographic orientation of the individual grains. As the strut is selected from an area which would correspond in a regular bulk sample to an $a$-growth sector, the dominating direction of the YBCO matrix is not [001], but shows a direction corresponding to the original location of the strut. So, the YBCO matrix exhibits mainly colors of orange and blueish-green (see Fig. 6(b)). In Ref. [27], it was pointed out that the orientation measurement by EBSD faces problems due to pseudosymmetry; that is, the analysing software may confuse $1 / 3$ of the $c$-axis of YBCO $(11.637 \AA$, $c / 3=3.88 \AA$ ) with an $a$ - or $b$-axis $(3.811 \AA$ and $3.879 \AA$, respectively). This problem can be solved by a subsequent data clean-up process of the data set using the OIM analysis software package, where a special function for analysing pseudosymmetry is provided. This treatment is applied to Fig. 6(b), yielding several big YBCO grains without a large scatter of the orientations.

In the present case, we observe that large 211 particles may alter the orientation of the YBCO matrix growth in a similar way as observed in Refs. $[14,15]$. 
These places are marked in Fig. 6(b) by arrows. All the 211 grains are surrounded by 1-pixel misoriented spots which are due to the height differences as revealed in the AFM topography images (Figs. 3 and 4). These data points have to be excluded from the following texture analysis.

Finally, Fig. 7 gives the EBSD-determined pole figures for YBCO and 211. As mentioned before, a cleaned data set with respect to pseudosymmetry effects is employed for this analysis. The resulting orientation of the YBCO matrix is well developed with a strong spot in the center of the [001] pole figure, and three more maxima are observed. For the 211 phase, we observe only some dominating orientations (only several maxima are visible in the pole figures). These maxima correspond to the best fits of the two different unit cells. Here, it is important to note that the scanned area of the foam cross-section $(10 \mu \mathrm{m} \times 10 \mu \mathrm{m})$ is too small to judge about the general orientation relation between 211 and YBCO, but it gives important information about the local grain configuration. EBSD analysis performed on a foam strut taken from position (3) yields in contrast pole figures with a much larger scatter of the 211 orientations, but also here some dominating orientations (maxima) can be found. This situation is similar to that found in the conventional, bulk melt-textured samples [14-17].

The resulting microstructure of the foam struts shows some remarkable features which need to be understood in detail in order to design an improved preparation route. A positive result is that the foam struts are completely converted into YBCO, everywhere in the foam. This enables the flow of strong supercurrents as demonstrated by the trapped field measurements of Ref. [6]. The large amount of residing 211 particles is remarkable as such microstructure was not observed in any other type of superconducting sample. However, the large 211 particles are not suitable as flux pinning sites in strong fields, but only at small applied fields [28]. The 211 particles filling the cracks in the YBCO matrix may have positive and negative effects depending on their size as the crack filling enables a current flow across the cracks, but the insulating character of larger 211 particles may also hinder the current flow. This problem could be eventually solved by adding Ag to the additional YBCO powder [29]. The STM topography image reveals further a large number of tiny 211 particles being embedded in the YBCO matrix, which is a promising result concerning the flux pinning (a)

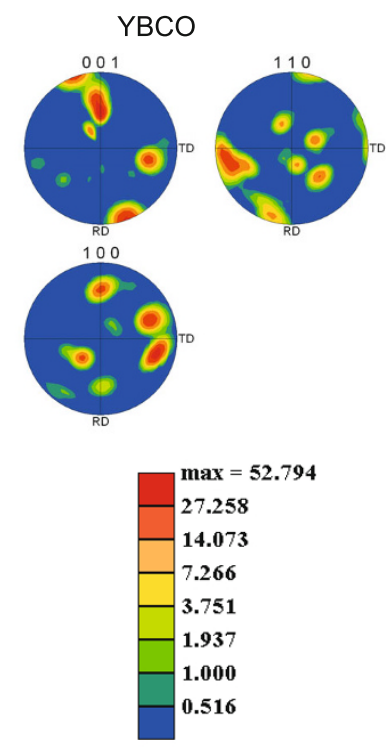

(b)

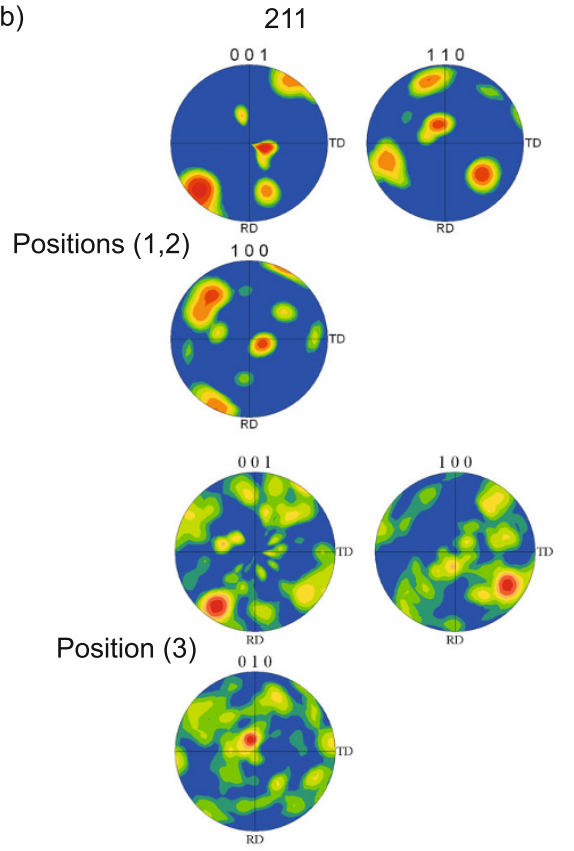

Fig. 7 EBSD pole figures for (a) YBCO and (b) 211 phases in [001], [100], [110] and [010]-directions. For the 211 particles, pole figures of positions (1), (2) and (3) are shown. The representation of the intensities of both phases is normalized, enabling a direct comparison. Here, one can see that at positions (1) and (2) for YBCO and 211 only some dominating directions exist, while the 211 phase in position (3) is much more randomly oriented. 
properties. The AFM/STM topography images further reveal that the superconducting matrix itself is built up of grains with dimensions of about $400 \mathrm{~nm} \times 200 \mathrm{~nm}$, which are clustered together. The clusters form then bigger blocks with dimensions in the micrometer range.

Nevertheless, there are some points where the microstructure of a YBCO foam could be further improved. The large number of relatively large 211 particles should be reduced, not only to obtain better suited flux pinning sites which should have a size in the $100 \mathrm{~nm}$ range, but also to reduce their negative influence on the orientation of the YBCO matrix. If these points can be achieved, the superconducting performance of these foams will considerably improve.

\section{Conclusions}

We have presented an analysis of the microstructure of the YBCO foams by means of AFM and EBSD. The measurements reveal that the struts are fully converted to the YBCO phase, and a large number of 211 particles are present. The 211 particles are randomly oriented, and the particle size ranges between $200 \mathrm{~nm}$ and $15 \mu \mathrm{m}$. The YBCO phase exhibits a large number of parallel-running cracks, some of which are filled with tiny 211 particles. Small 211 particles with sizes between $200 \mathrm{~nm}$ and $500 \mathrm{~nm}$ are found embedded in the YBCO matrix, which is useful for the flux pinning properties. Furthermore, the microstructure of the foam is remarkably similar in all locations investigated here. From our microstructure investigation, some important conclusions for the further improvement of such foams can be drawn.

\section{Acknowledgements}

We acknowledge collaborations within the European Forum for Processors of Bulk Superconductors (EFFORT), which is funded by the Engineering and Physical Sciences Research Council (EPSRC) of the UK government. We thank M. Winter (Saarland University) for his efforts with the AFM measurements. The 3D-images were prepared using the image processing software described in Ref. [30].

Open Access: This article is distributed under the terms of the Creative Commons Attribution License which permits any use, distribution, and reproduction in any medium, provided the original author(s) and the source are credited.

\section{References}

[1] Reddy ES, Schmitz GJ. Superconducting foams. Supercond Sci Technol 2002, 15: L21-L24.

[2] Reddy ES, Schmitz GJ. Ceramic foams. Am Ceram Soc Bull 2002, 81: 35-37.

[3] Noudem JG, Reddy ES, Schmitz GJ. Magnetic and transport properties of $\mathrm{YBa}_{2} \mathrm{Cu}_{3} \mathrm{O}_{y}$ superconductor foams. Physica C 2003, 390: 286-290.

[4] Reddy ES, Herweg M, Schmitz GJ. Processing of $\mathrm{Y}_{2} \mathrm{BaCuO}_{5}$ foams. Supercond Sci Technol 2003, 16: 608-612.

[5] Noudem JG, Guilmeau E, Chateigner D, et al. Properties of $\mathrm{YBa}_{2} \mathrm{Cu}_{3} \mathrm{O}_{y}$-textured superconductor foams. Physica C 2004, 408-410: 655-656.

[6] Bartolomé E, Granados X, Puig T, et al. Critical state in superconducting single-crystalline $\mathrm{YBa}_{2} \mathrm{Cu}_{3} \mathrm{O}_{7}$ foams: Local versus long-range currents. Phys $\operatorname{Rev} B$ 2004, 70: 144514.

[7] Masson PJ, Brown GV, Soban DS, et al. HTS machines as enabling technology for all-electric airborne vehicles. Supercond Sci Technol 2007, 20: 748-756.

[8] Mitschang GW. Space applications and implications of high temperature superconductivity. IEEE T Appl Supercon 1995, 5: 69-73.

[9] Gu C, Zou S, Qu T, et al. Design of HTS coil for magnetic driving spacecraft. IEEE T Appl Supercon 2010, 20: 997-1000.

[10] Jung A, Diebels S, Koblischka-Veneva A, et al. Microstructural analysis of electrochemical coated open-cell metal foams by EBSD and nanoindentation. Adv Eng Mater 2014, 16: 15-20.

[11] Fiertek P, Andrzejewski B, Sadowski W. Synthesis and transport properties of porous superconducting ceramics of $\mathrm{YBa}_{2} \mathrm{Cu}_{3} \mathrm{O}_{7-\delta}$. Rev Adv Mater Sci 2010, 23: $52-56$.

[12] Noudem JG. Development of shaping textured $\mathrm{YBaCuO}$ superconductors. J Supercond Nov Magn 2011, 24: 105-110.

[13] Diko P, Kaňuchová M, Chaud X, et al. Oxygenation mechanism of TSMG YBCO bulk superconductor. J Phys: Conf Ser 2008, 97: 012160.

[14] Koblischka-Veneva A, Koblischka MR, Mücklich F, et al. Interactions of $\mathrm{Y}_{2} \mathrm{BaCuO}_{5}$ particles and the YBCO matrix within melt-textured YBCO samples studied by means of electron backscatter diffraction. Supercond Sci Technol 2005, 18: S158-S163.

[15] Koblischka-Veneva A, Koblischka MR, Ogasawara 
$\mathrm{K}$, et al. Interplay of YBCO and embedded 211 particles in melt-textured YBCO superconductors. J Supercond 2005, 18: 469-474.

[16] Guilmeau E, Lambert S, Chateigner D, et al. Quantitative texture analysis of polyphased oxides by diffraction: Example of Bi2223 sinter-forged cearmic and Y123 foam superconductors. Mat Sci Eng B 2003, 104: 107-112.

[17] Chateigner D, Wenk H-R, Pernet M. Orientation analysis of bulk YBCO from incomplete neutron diffraction data. J Appl Cryst 1997, 30: 43-48.

[18] Humphreys FJ. Characterisation of fine-scale microstructures by electron backscatter diffraction (EBSD). Scripta Mater 2004, 51: 771-776.

[19] Koblischka-Veneva A, Mücklich F, Koblischka MR, et al. Crystallographic orientation of $\mathrm{Y}_{2} \mathrm{Ba}_{4} \mathrm{CuMO}_{x}$ $(\mathrm{M}=\mathrm{Nb}, \mathrm{Zr}, \mathrm{Ag})$ nanoparticles embedded in bulk, melt-textured YBCO studied by EBSD. J Am Ceram Soc 2007, 90: 2582-2588.

[20] Struers. Metalog guide. Available at http://www. testinginstrument.com/cgi-bin/images/001192_pdf. pdf.

[21] Koblischka-Veneva A, Koblischka MR. Surface preparation of high- $T_{\mathrm{c}}$ superconductors for $\mathrm{MO}$ imaging. In Magneto-Optical Imaging. Johansen TH, Shantsev DV, Eds. Dordrecht: Kluwer Acad Press, 2004: 242-246.

[22] Koblischka MR, Winter M, Hu A, et al. Stripe and criss-cross patterns in high- $T_{\mathrm{c}}$ superconductors revealed by atomic force microscopy and scanning tunnelling microscopy. Jpn J Appl Phys 2006, 45:
2259-2263.

[23] Winter M, Koblischka MR, Koblischka-Veneva A, et al. Nano-stripe structures in light rare-earth high- $T_{\mathrm{c}}$ superconductors. J Phys: Conf Ser 2007, 61: 601-605.

[24] TexSEM Laboratories (TSL). Orientation imaging microscopy software version V4.0, user manual. TexSEM Laboratories (TSL), Draper, UT, USA.

[25] Koblischka-Veneva A, Koblischka MR, Simon P, et al. Electron backscatter diffraction study of polycrystalline $\mathrm{YBa}_{2} \mathrm{Cu}_{3} \mathrm{O}_{7-\delta}$ ceramics. Physica $C$ 2002, 382: 311-322.

[26] Koblischka MR, Koblischka-Veneva A. Applications of the electron backscatter diffraction technique to ceramic materials. Phase Transit 2013, 86: 651-660.

[27] Grossin D, Henrist C, Mathieu J-Ph, et al. EBSD study on YBCO textured bulk samples: Correlation between crystal growth and 'microtexture'. Supercond Sci Technol 2006, 19: 190-199.

[28] Jirsa M, Zablotskii V, Petrenko P, et al. Vortex interaction with large particles in bulk RE-123 compounds. J Low Temp Phys 2005, 139: 103-109.

[29] Obradors X, Yu R, Sandiumenge F, et al. Directional solidification of $\mathrm{REBa}_{2} \mathrm{Cu}_{3} \mathrm{O}_{7} \quad(\mathrm{RE}=\mathrm{Y}, \quad \mathrm{Nd})$ : Microstructure and superconducting properties. Supercond Sci Technol 1997, 10: 884-890.

[30] Horcas I, Fernández R, Gómez-Rodríguez JM, et al. WSXM: A software for scanning probe microscopy and a tool for nanotechnology. Rev Sci Instrum 2007, 78: 013705. 